\title{
Mathematical Model of Aedes Outbreaks in Padang City Considering Climate Parameter
}

\author{
Efendi ${ }^{1}$, Jenizon¹, Budi Rahmadya ${ }^{2}$, and M. Hafiz Hersyah ${ }^{2}$ \\ ${ }^{1}$ Andalas University, Faculty of Science, Mathematics Department, Limau Manis, Padang, \\ Indonesia, 25163 \\ ${ }^{2}$ Andalas University, Faculty of Informatics Technology, Computer System Department, Limau \\ Manis, Padang, Indonesia, 25163
}

\section{Abstract}

Basic reproduction number is a threshold number that frequently found in the analysis of determining the spreads of population. Many methods have been derived to calculate basic reproduction number including eigen values or radius spectral and statistical approximation. However, the calculation of the threshold number is still not easy and tends to use a lot of computation. Another approach has been proposed using graph reduction. This approach has been applied to determine threshold number

Corresponding Author:

Efendi

efendi97unand@gmail.com

Received: 19 February 2019

Accepted: 5 March 2019

Published: 16 April 2019

Publishing services provided by

Knowledge E

(c) Efendi et al. This article is distributed under the terms of the Creative Commons

Attribution License, which permits unrestricted use and redistribution provided that the original author and source are credited.

Selection and Peer-review under the responsibility of the ICBSA Conference Committee.

\section{G OPEN ACCESS} of Aedes spreads in Padang city depending on climate parameter. The daily rainfall data is used as an empirical study.

Keywords: basic reproduction number, population projection, graph reduction, climate parameters

\section{Introduction}

Padang like other city in Indonesia has tropical climate. Its climate is hot, humid weather along the year (see Fig. 1). This condition results in Padang as endemic for vector-borne diseases including dengue fever (DF) that transmitted by Aedes aegypti and Aedes albopictus. It is known that DF first emergence is in Jakarta and Surabaya around 1968. Since then DF and its more severe form, dengue hemorrhagic fever (DHF), have been growing steadily to a significant high incidence rate $[1,2]$. Nowadays, the changing patterns of dengue epidemiology have been recognized as a challenge for control and have made it difficult to understanding for scientist and stakeholder. [3]

One method to understand the epidemic diseases and to analyze strategies to control the transmission is mathematical modelling. Many research have been done using this method, the early one was done by Kermack and Mc. Kendrick that succesfully developed an SIR model to describe epidemic diseases in 1927 [4]. The SIR model then had some modification like SEIR, MSIR etc, to know the transmission dynamics of infectious 


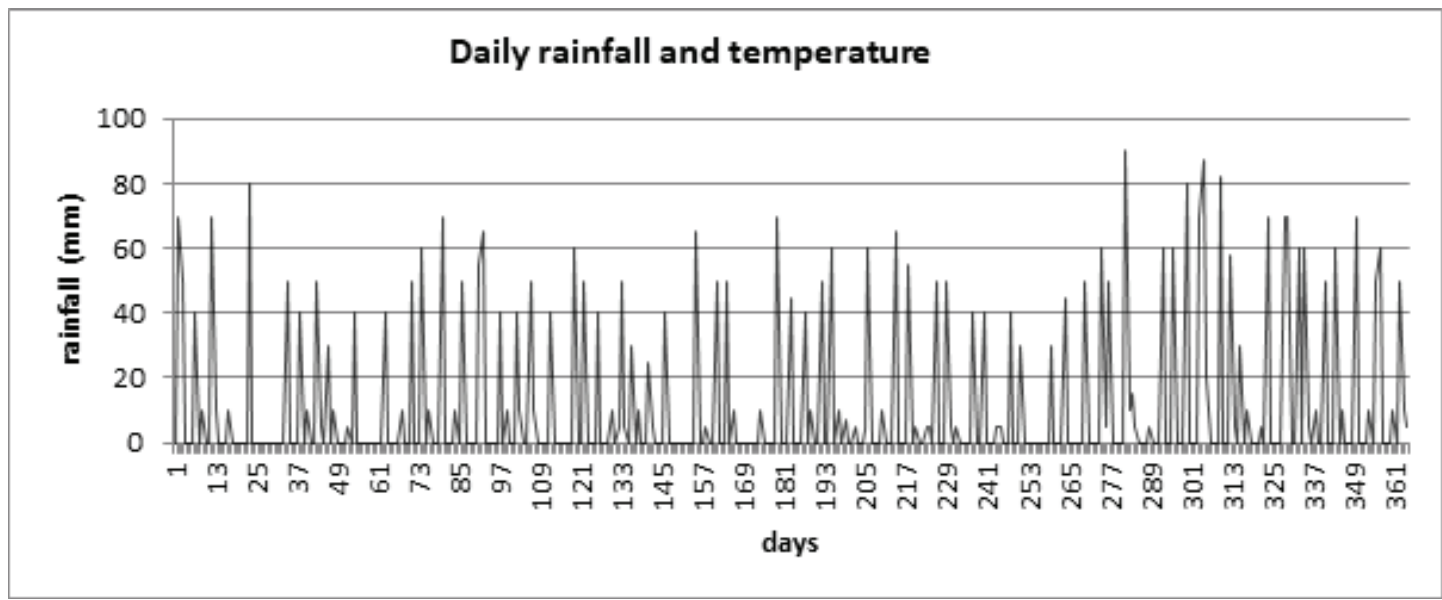

Figure 1: Daily rainfall and temperature in Padang during 2017 (source BMKG Padang).

diseases. Another tried to address transmission considering human population as a variable $[5,6]$.

Dengue outbreaks are sensitive to the climate. It can be seen from the variety of cases of DF in Padang, that are 103 cases in January, 137 in February, 130 in March, 105 in April, 83 in May, 60 in June, 35 in July and 53 in Agust. Changing climate factors also affect the geographically spreadout and future dengue projections. One principal of Aedes mosquitoes' survival is temperature due to seasonal changes. The temperature plays an important role in the life cycle and behavior of the mosquitoes. Aedes survival at different temperature levels in laboratory and field settings have been reported by Bradly et al. [10]. Therefore, in this present work, we propose a mathematical model of Aedes outbreaks in Padang city considering climate parameter. We have considered rainfall and temperature in Padang along 2017-2018 that control the transision of female Aedes to each class of age-structured population.

\section{Materials and Methods}

\subsection{Materials}

Materials used in this paper are mathematics formulas that describe fluctuation of Aedes outbreaks in Equation. The equation are constructed in matrix projection with entry means transition of each stage of mosquito population, For the needs, it is assumed that the life cycle of Aedes start with three water-dependent stages in form of egg, larva, and pupa, and last with aerial stage as adult mosquito. The cycle is quiet similar to all mosquito species. Adult mosquito can be differed to two stages due to the ability to egg hatching. As the water availability dependence, the lifespan transition is also 
depending on temperature. With these climate effects, the life spans end with female adults that seek a human host to take a blood meal. The female then rest in a sheltered place during the few days needed for the eggs to mature. After that the female search for sites to lay their eggs. The female mosquito breeds in artificial containers of any type. Usually, the breeding is in small natural water bodies such as tree holes or rock pools [10]. Eggs mosquito then hatch after a desiccation period that can be ranged from few days to several months. The desiccation is effected by water availability that accumulated from rainfall or artificial flooding. The larvae then mature through three stages before entering pupation. Adult mosquito emerges from the pupa at the surface of water. In temperate climates, Aedes survive the unfavorable period (winter) as eggs in dormancy (diapauses) that will hatch during the next favorable season (spring).

Area of study is in Padang city where the climate is typically hot/warm, very dry summers and mild, wet rainy. Total annual rainfall is around $750 \mathrm{~mm}$, and temperatures usually vary between $20^{\circ} \mathrm{C}$ to $34^{\circ} \mathrm{C}$. Aedes populations are remaining exists in these areas.

For understanding the dynamic of the abundance of mosquito we use Model of Aedes Abundance using matrix population projection with each entry of the matrix represent the transition that depended on climate parameter.

\subsection{Methods}

The generic model of Aedes dynamics developed in this paper represents all of the steps of the life cycle. It is considered five different stages: three aquatic stages (A,B eggs; L, larvae). two emerging adult stage. In the adult stage, females only are represented. Parous females are females that have oviposited at least once, whereas nulliparous females have never laid eggs. Adults are subdivided regarding their behaviour during the gonotrophic cycle (h, host-seeking; g, transition from engorged to gravid; o, oviposition site seeking). Once parous, females repeat their gonotrophic cycle until death. The events driving the transitions between stages are: egg mortality or hatching, larva mortality, pupation (moult of larvae to pupae), pupa mortality, adult emergence, mortality, engorgement, egg maturing, and oviposition. The model takes into account density-dependent mortality of the larval stage, and pupa density-dependent success of adult emergence. Density-dependent mortality was assumed at the larval stage as it is has been often observed. With compartmental model, we should compute basic reproduction number to show the relationship between rates of transition of each compartment with the abundance of Aedes. After having an understanding the relations 
mathematically we use simulation to conduct with climate parameter effects on Aedes population. The simulation will show the abundance of Aedes in Padang and we will also compare it with dengue fever (DF) occurring in Padang to have a validation of the model. For the analysis we use basic reproduction number to determine the dynamics of Aedes abundance in constant rate of transition. The basic reproduction number is computed using graph reduction method. For climate dependent model, the simulation is drawn to explain the dynamics of the Aedes outbreaks.

\section{Results and Discussions}

Let the compartments of female Aedes being named by $A, B, L, C$, and $D$. Let $A=$ newly egg,B=mature egg, $\mathrm{L}=\frac{\text { Larvae }}{\text { pupae }}, \mathrm{C}=$ =early adult, $\mathrm{D}=$ mature, a $q_{i j}=$ transition rate, $q_{i M}=$ mortality rate, and $F_{C}, F_{D}$ = fecundity. The model of dynamics of Aedes is drawn in digraph in Fig. 2.

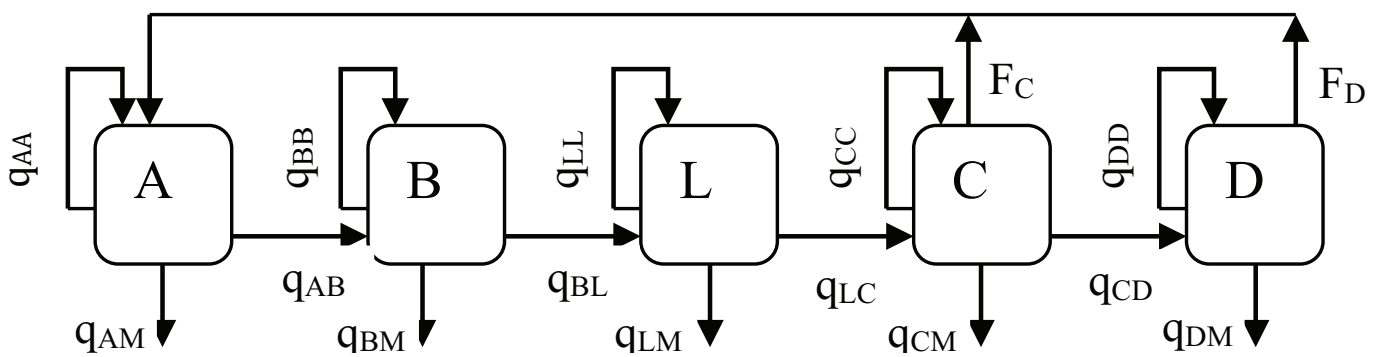

Figure 2: Age-structured model of female Aedes abundance.

Using the digraph, we can construct transition matrix for population projection. The transition matrix $Q$ is defined as Eq. (1):

$$
Q=\left(\begin{array}{ccccc}
q_{A A} & 0 & 0 & F_{C} & F_{D} \\
q_{A B} & q_{B B} & 0 & 0 & 0 \\
0 & q_{B L} & q_{L L} & 0 & 0 \\
0 & 0 & q_{L C} & q_{C C} & 0 \\
0 & 0 & 0 & q_{C D} & q_{D D}
\end{array}\right)
$$

Therefore the abundance of stage vector of Aedes at time t can be written as Eq. (2):

$$
N_{t}=Q \times N_{t-1}
$$

with:

$$
N_{t}=\left(A_{t}, B_{t}, L_{t}, C_{t}, D_{t}\right)^{T}
$$




\subsection{Computation of basic reproduction number}

To compute basic reproduction number (Ro) as suggest by [1] and [2], the simplifying age-structured model should be redrawn as Fig. 3, by removing all mortality rate and change the symbol of transition rate for convenience.

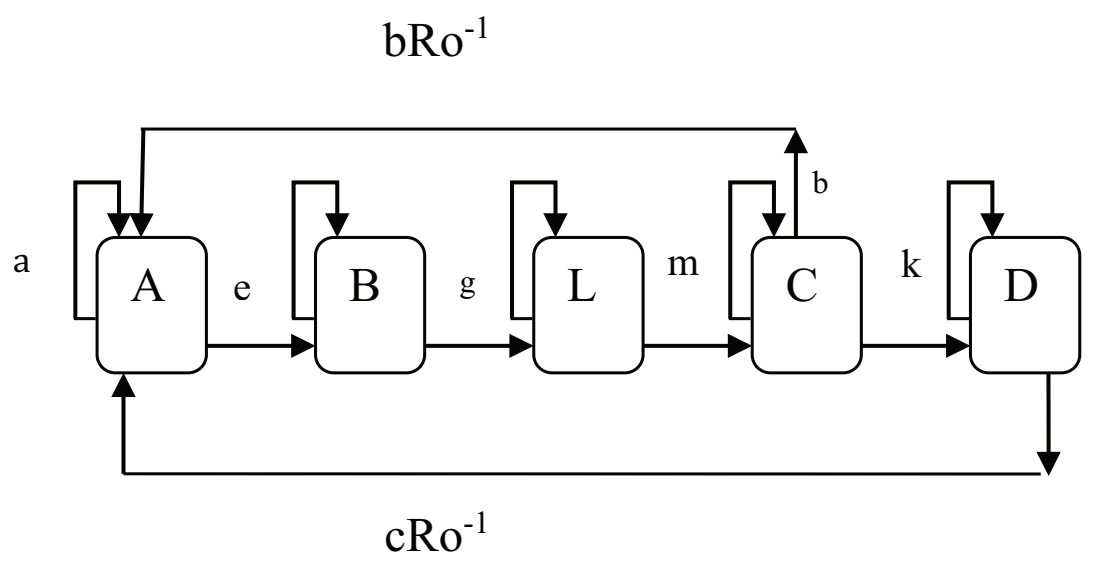

Figure 3: The digraph of simplifying age-structured model.

The computation of basic reproduction number ( $R o$ ) is by removing all cycle of simplifying digraph, so that the graph can be drawn as Fig. 4 .

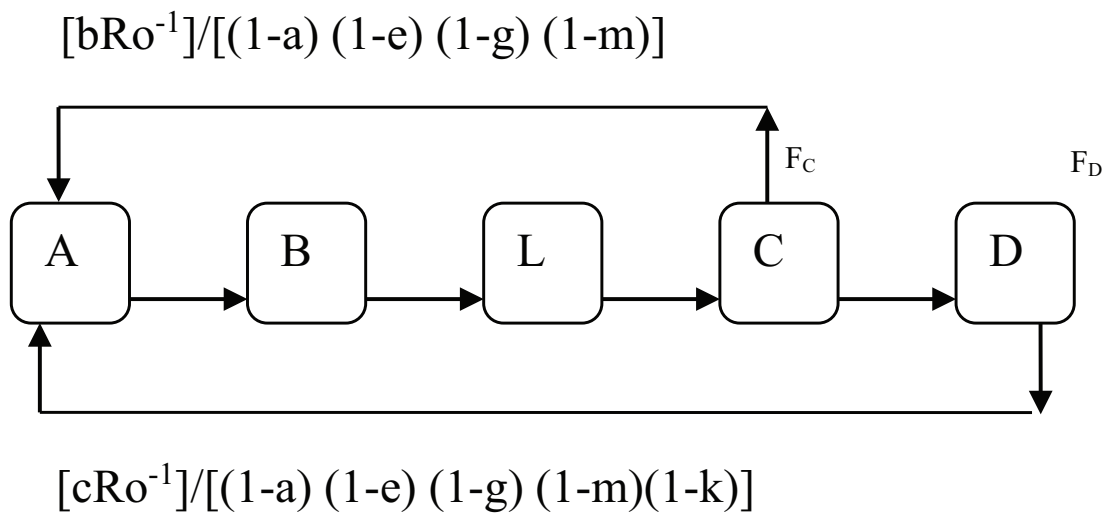

Figure 4: The digraph without cycle.

By removing state $B$ and $L$, then the digraph can be drawn as Fig. 5 .

By removing state $D$, then the digraph can be drawn as Fig. 6 .

At last, by removing state $A$, then the digraph can be drawn as Fig. 7, as a final graph reduction. 


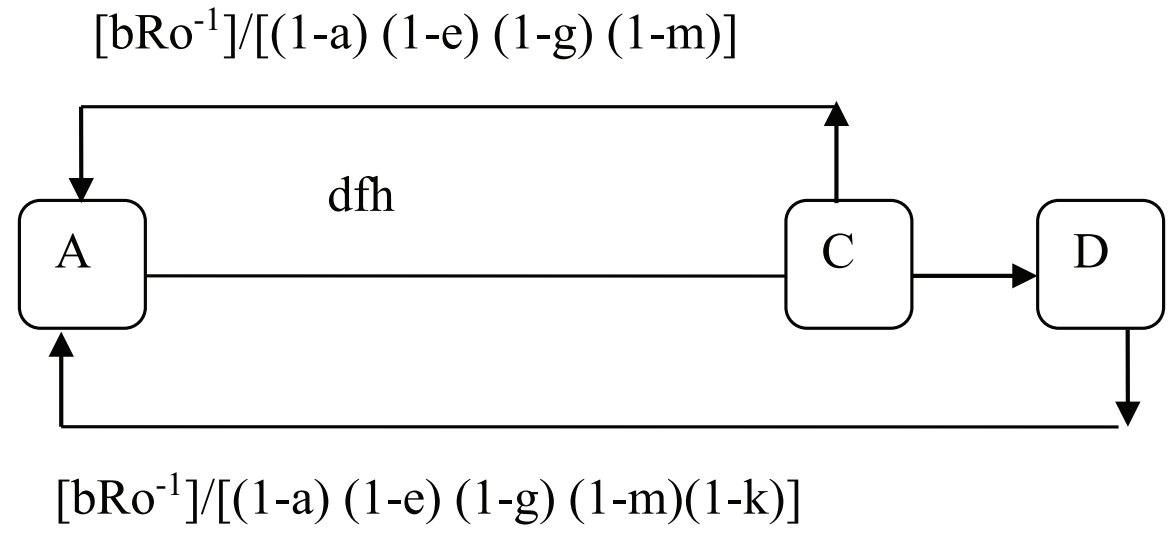

Figure 5: The digraph without state B and L.

$\left[\mathrm{bRo}^{-1}\right] /[(1-\mathrm{a})(1-\mathrm{e})(1-\mathrm{g})(1-\mathrm{m})]$

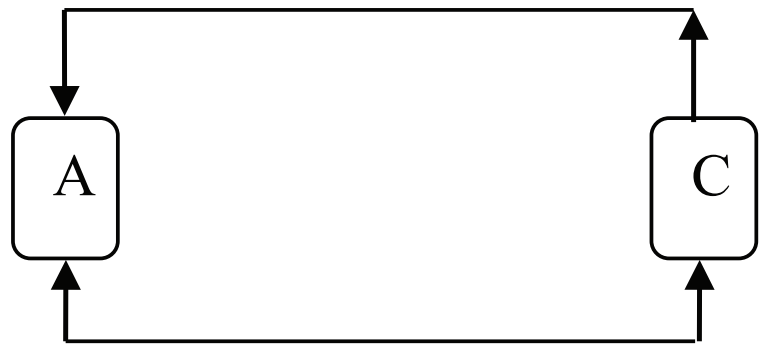

$$
\left[\mathrm{ncRo}^{-1}\right] /[(1-\mathrm{a})(1-\mathrm{e})(1-\mathrm{g})(1-\mathrm{m})(1-\mathrm{k})]
$$

Figure 6: The digraph without state B and L.

$$
\left[\mathrm{dfhbRo}{ }^{-1}\right] /[(1-\mathrm{a})(1-\mathrm{e})(1-\mathrm{g})(1-\mathrm{m})]
$$

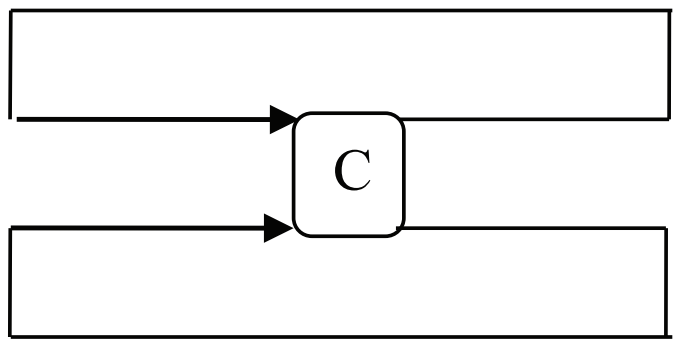

$$
\left[\mathrm{dfhbRo}{ }^{-1}\right] /[(1-\mathrm{a})(1-\mathrm{e})(1-\mathrm{g})(1-\mathrm{m})]
$$

Figure 7: Final digraph reduction. 
Based on Fig. 7, the basic reproduction number should satisfy total as shown in Eq. (3):

$$
\frac{\left[d f h b R_{0}^{-1}\right]}{[(1-a)(1-e)(1-g)(1-m)]}+\frac{\left[d f h n c R_{0}^{-1}\right]}{[(1-a)(1-e)(1-g)(1-k)]}=1
$$

By solving Eq. 1 for Ro (Eq. (4)), then:

$$
R_{0}=\frac{[d f h(b(1-k)+c n)]}{[(1-a)(1-e)(1-g)(1-m)(1-k)]}
$$

Using Eq. (4), the simulation of Aedes abundance with Ro $=1,296>1$ is shown in Fig. 8 .

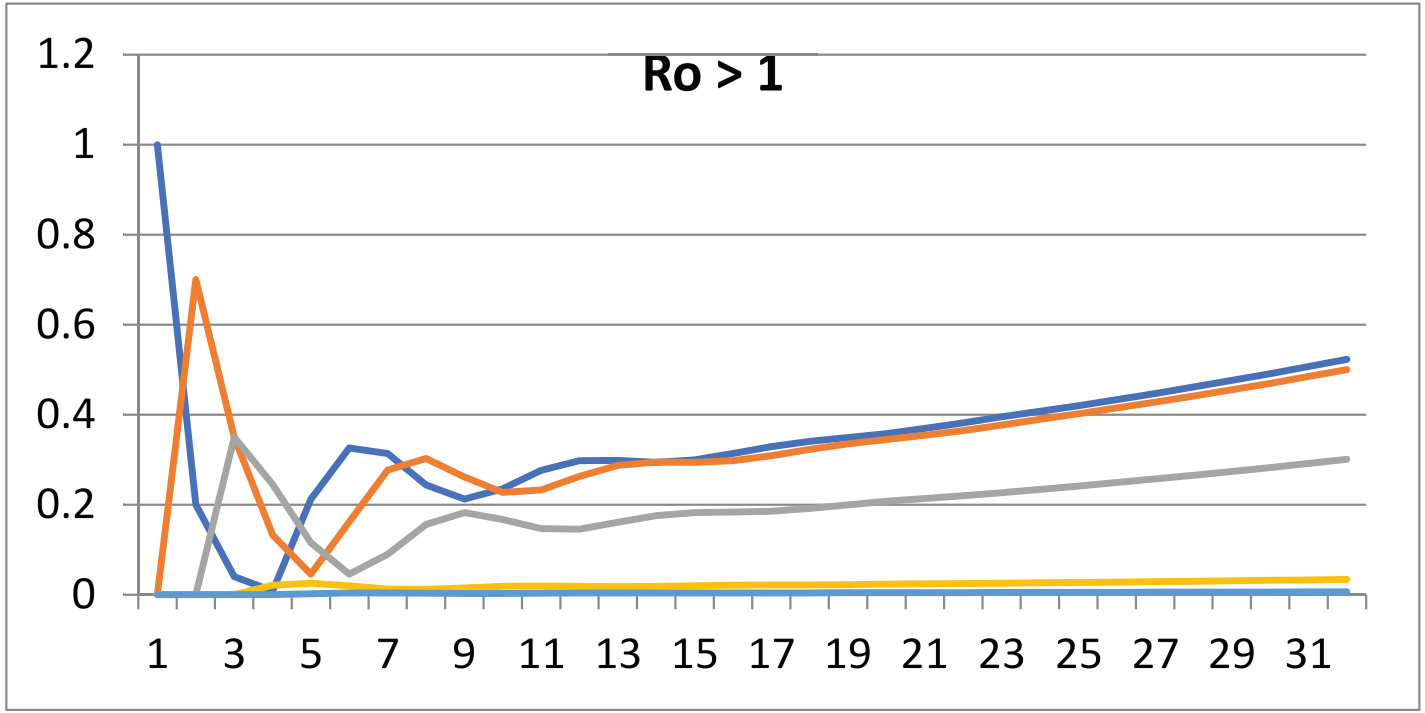

Figure 8: Population dynamics due to Ro.

The simulation for constant transition in Fig. 8, without climate effect shows that, the abundance of adult mosquito will increase to infinity if RO (basic reproduction number) in Eq. (4) is larger than one. The RO will depend on each state transition as also concluded by [5], [6], [7], [8], and [9]. As indicated by Eq. (4), Ro will proportional to rate of b, c, d, $\mathrm{f}$ and $\mathrm{h}$, it means if these rate increase than $\mathrm{RO}$ will also increase. On the otherhand, $\mathrm{RO}$ is proportional to the inverse of $\mathrm{a}, \mathrm{e}, \mathrm{g}, \mathrm{m}$, and $\mathrm{k}$ that represents the rate of being stayed at the same stage after period of transition. It means that, if we can make some conditions such that the transition of mosquito in each stage are not running well, than we can hope that we can reduce the adults mosquito and with this assumption we can reduce DF occuring in Padang.

\subsection{Model with climate effect}

The shape of habitat of Aedes is assumed to be cylinder because of the factual finding in field that the habitat of aedes is usually the used can, tree hole etc. In the cylinder 
the dynamic of water availability can be viewed in Fig. 9. Let EPmax be the maximal capacity of breeeding sites with depth $\mathrm{H}$ and radius $\mathrm{R}$, then $\mathrm{EPmax}=\pi R^{2} H$. Consider water dynamics in man made container with cylinder shape as shown in Fig. 9.

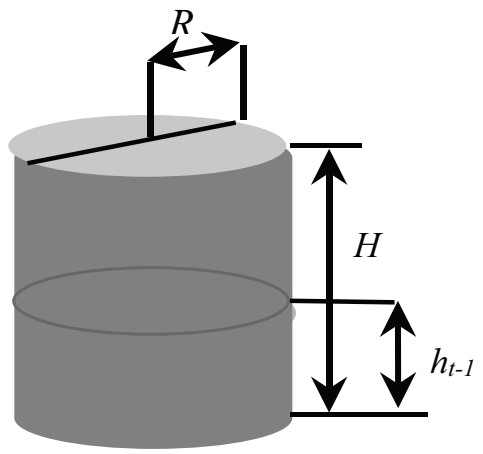

(a)
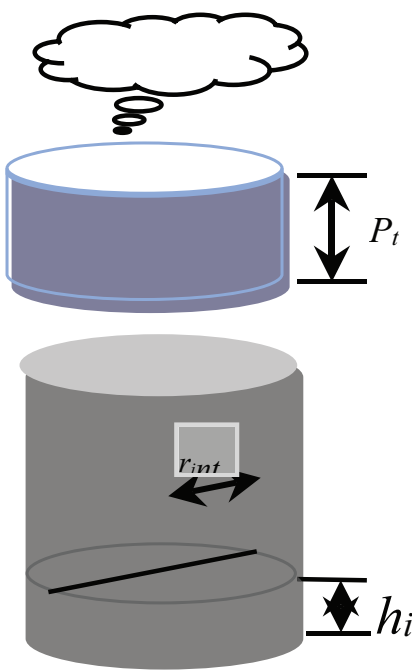

(h)

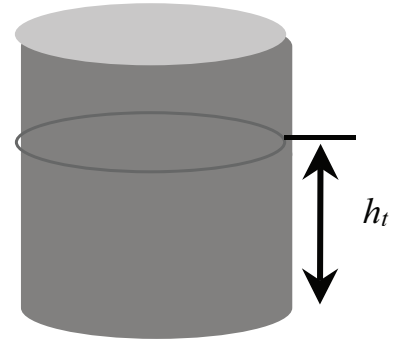

(c)

Figure 9: Water dynamics in cylinder. (a) time t-1 (b) time for infiltrasion with rain and evaporation (c) time t.

Water from the rain infiltrate to the cylinder and EPt is being updated. The water in cylinder is lose because of evaporation. Between $\mathrm{t}-1$ and $\mathrm{t}$ the evaporation is $\theta_{\text {evap }, t} E P_{t-1}$ where $\theta_{\text {evap }, t}$ is evaporation rate in Eq. (5). So that the water that available in (t-1,1) is $\left(1-\theta_{\text {evap }, t}\right) E P_{t-1}$. This rate depends on surface area $S_{t-1}=\pi\left(r_{i n t}\right)^{2}=\pi(R)^{2}$. So that the evaporation rate can be chosen as a monoton function:

$$
\theta_{\text {evap }, t}=1-e^{-\rho_{\text {evap }} S_{t-1}}=1-e^{-\rho_{\text {evap }} \pi R^{2}} ; \quad \rho_{\text {evap }} \geq 0
$$

Let EPint is the amount of water after evaporationat $t-1$ to $t$, then

$$
E P_{\text {int }}=E P_{t-1}\left(1-e^{-\rho_{\text {evap }} \pi R^{2}}\right)
$$

If it is rain with rainfall $\mathrm{Pt}$, the water rain come into cylinder with depth Pt.. Using Eq. (5) and Eq. (6), the final volume of water in cylinder after evaporation and rain water entrance is:

$$
E P_{t}=E P_{\text {int }}+\left(\theta_{\text {eff }, t} \pi R^{2} P_{t}\right)=E P_{t-1}\left(1-e^{-\rho_{\text {evap }} \pi R^{2}}\right)+\pi R^{2} P_{t}
$$

With Eq. (7), the maximum capacity of cylinder EPmax, then the final volume at time $t$ will be shown in Eq. (8):

$$
E P_{t}=\min \left(E P_{t-1}\left(1-e^{-\rho_{\text {evap }} \pi R^{2}}\right)+\pi R^{2} P_{t}, \quad \text { EPmax }\right)
$$




\subsection{Environmental data}

Daily rainfall and temperature data from January 2017 to August 2018 recorded Padang were obtained from the national meteorological service (BMKG). We assumed that the population dynamics of Aedes is mainly driven by these two factors: (i) temperatures have a strong impact on the survival of Ae. albopictus populations, and on the development of aquatic stages [10]; (ii) precipitations favor the availability of breeding sites, i.e., any small recipient filled with water where Ae. albopictus females lay their eggs. Moreover, we considered that the egg hatching is triggered by rainfall events but also by human water supply. Indeed, larval surveys carried out, showed the importance of small and medium containers sampled in gardens in the productivity Ae. albopictus' populations. For the need of simulation, the parameter of biology, habitat and climate for Aedes are shown Table 1 and Table 2.

TABLE 1: Biology and habitat parameter of Aedes [10].

\begin{tabular}{l|c|c|} 
& Parameter & A.aegepty \\
\hline Biological parameter & & \\
\hline Time in stage A (days) & $T_{A}$ & 7 \\
\hline Mortality rate in A & $q A M$ & 0.01 \\
\hline Mortality rate in B & $q B M$ & 0.01 \\
\hline Time in stage L (hari) & $T_{B}$ & 16 \\
\hline Mortality rate in L & $q L M$ & 0.01 \\
\hline Hatching period & $T C$ & 7 \\
\hline Mortality rate in C & $q C M$ & 0.01 \\
\hline Mortality rate in D & $q D M$ & 0.06 \\
\hline Total Egg/female & Nwf & 300 \\
\hline Habitat parameter & & \\
\hline Egg laying depth $(m m)$ & $\mathrm{H}$ & 80 \\
\hline Habitat Radius & $\mathrm{R}$ & 30 \\
\hline Simulation Asumption & & \\
\hline Beginning population & $\mathrm{NO}$ & $(0,0,0,0,1)$ \\
\hline Time step (days) & $\Delta \mathrm{t}$ & 1 \\
\hline
\end{tabular}

With some parameters are time dependent for the sake of climate parameter. Considering the climate parameter effect, the transition rate will depend on time step $t$. We assume only stage $A, B$ and $L$ that being depended on time step $t$ because of the 
TABLE 2: Estimated parameter [10].

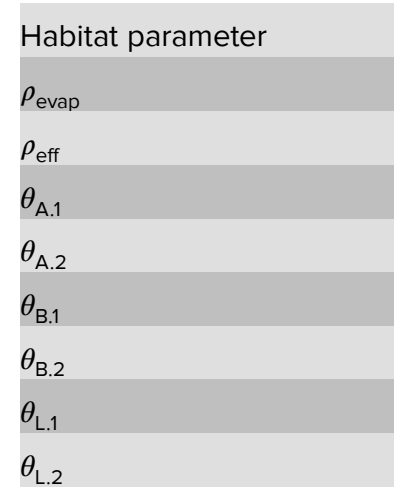

\begin{tabular}{|c|}
\hline A.aegepty \\
\hline 0.00005 \\
2.27 \\
- \\
- \\
0.03 \\
0.67 \\
0.25 \\
0.28
\end{tabular}

maturity of the egg need a period of dry an wet. In this condition, the transition matrix $Q$ can be written as Eq. (9).

$$
Q_{t}=\left(\begin{array}{ccccc}
q_{A A, t} & 0 & 0 & F_{C} & F_{D} \\
q_{A B, t} & q_{B B, t} & 0 & 0 & 0 \\
0 & q_{B L, t} & q_{L L, t} & 0 & 0 \\
0 & 0 & q_{L C, t} & q_{C C} & 0 \\
0 & 0 & 0 & q_{C D} & q_{D D}
\end{array}\right)
$$

Therefore the abundance of stage vector of Aedes at time t can be written as Eq. (10).

$$
N_{t}=Q_{t} \times N_{t-1}
$$

The transition parameters depend on fluctuation of water in breeding sites and for transition parameters functions we use as listing in [10] and using Eq. (8), the simulation will have an information about the climate effect.

\subsection{Simulation}

With parameters and functions listed above, the simulation results as shown in Fig. 10. The dynamics of Aedes in Padang area present strong climate variability with 6 peaks adult abundance outbreaks. The first eggs of the year are laid at the beginning of November 2017. Mosquito density is maximal early January to February 2018. The dynamics continues to August and early September 2018. Oviposition activity decreases between mid-September and mid-October, with a residual egg laying activity remaining until November or December. Sampling results did not show evidence of continuous oviposition activity during sunny, Based on observed temperatures and precipitations from 2017 to 2018, the model showed Aedes adult mosquitoes to be present along 
these years with a maximum population in February 2018 (Fig. 10). The number of eggs reaches a maximum at the beginning of the unfavorable period, when eggs stop hatching while adult females continue oviposition activity. The egg reserve decreases during winter time, allowing the survival of the population. Differences between years were due to differences in weather variables, the model being otherwise deterministic.

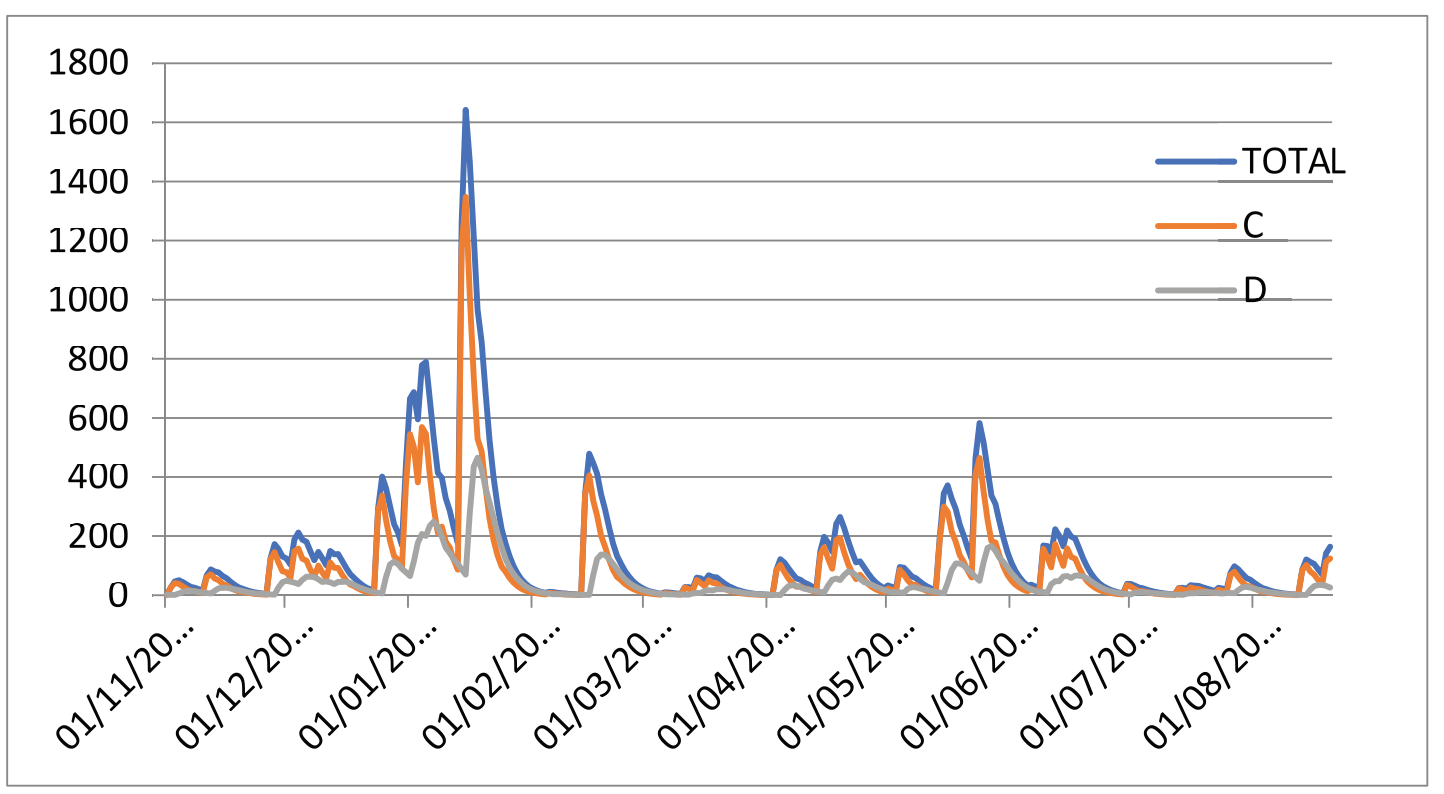

Figure 10: The abundance of adult Aedes from 01/11/2017-20/08/2018.

As a comparansion the reported cases in Padang, that there are 103 cases in January, 137 in February, 130 in March, 105 in April, 83 in May, 60 in June, 35 in July and 53 in Agust, the plot of these cases can be seen in Fig. 11. Simulated mosquito abundances were quiet consistent with field data. For the one year under consideration the model reproduces well the abundance peak of Dengue Fever occurring in 2017-2018. For both years the model simulates well the population growth in March to September. Yet, the model overestimates the abundances of Aedes at January 2018.

\section{Conclusions}

Climate parameter are sensitive to Dengue outbreaks. We have considered Aedes mosquitoes survival in seasonal changes of temperature and rainfall. The temperature plays an important role in the life cycle and behavior of the mosquitoes. The effect of climate parameter to Aedes were applied to projections model in stage egg and larvae. The sensitive of the parameter should be study to have a better understanding of climate effect. On the otherhand, RO is proportional to the inverse of the rate of being stayed at the same stage after period of transition. It means that, if we can make some 


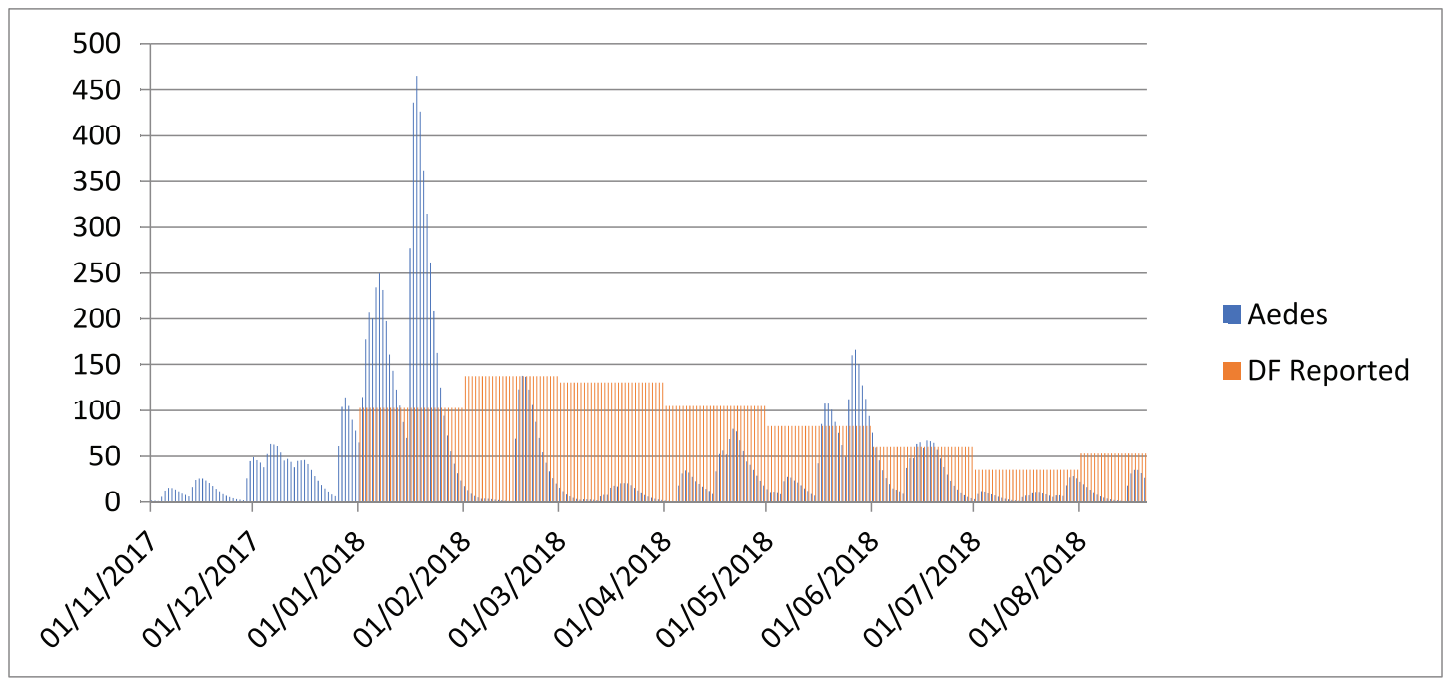

Figure 11: Abundance of adult Aedes and reported cases of DS in Padang.

conditions such that the transition of mosquito in each stage are not running well, than we can hope that we can reduce the adults mosquito and with this assumption we can reduce DF occuring in Padang. These can be done by maintaining the habitat of Aedes leak from dynamic of water availabity or the best practice is by removing the habitat of Aedes population.

\section{Acknowledgments}

This research is accomplished with BOPTN funding. We thank to Andalas University for opportunity to conduct this research. We also thank to lecture of Mathematics Department for helpful discussions and comments on the manuscript.

\section{References}

[1] de-Camino-Beck T., Lewis, M.A. (2007). A New Method for Calculating Net Reproductive Rate from Graph Reduction with Application to The Control of Invasive Species. Bull. Math. Biology. 69(4):1341-1354.

[2] de-Camino-Beck T., Lewis, M.A. (2008). A Graph Theoritic Method for Basic Reproduction Number in Continous Time Epidemiological Models. Bull. Math. Biology. 172(1):128-139.

[3] Caswell, H. (2001) Matrix Population Models: Construction, Analysis, and Interpretation. 2nd ed. Sinauer Ass. USA. 
[4] Cruz G.P., Esteva L., Montano J.A., Vargas C., (2004). A Mathematical Model for The Dynamics of West Nile Virus.

[5] Driessche, V.D., Watmough, J. (2002). Reproduction Numbers and Subthreshold Endemic Equilibria for Compartmental Models of Disease Transmission. Math. Biosci. 180:29-48.

[6] Heesterbeek, J. (2002). A Brief History of RO and a Recipe for Its Calculation. Acta Biotheoretica 50:189-204.

[7] Lewis, M.A., Reclawowicz, Driessche, V.D. Wonham M. (2006). A comparison of Continous and Discrete-time West Nile Virus model. Bull. Math. Bio.491-509.

[8] Mason, S., Zimmermann, H. (1960). Electronic Circuits,Signals, and Systems. Wiley, New York.

[9] Supriatna, A.K. (2009) Estimating the basic reproduction number of dengue transmission during 2002-2007 outbreaks in Bandung, Indonesia. Dengue Bulletin Vol.33.

[10] Schaeffer B, Mondet B, Touzeau S. (2008) Using a climate-dependent model to predict mosquito abundance: application to Aedes (Stegomyia) africanus and Aedes (Diceromyia) furcifer (Diptera: Culicidae), Infection, Genetics and Evolution Vol. 8, Issue 4. 